\title{
MedienPädagogik
}

www. medienpaed.com

Zeitschrift für

Theorie und Praxis

der Medienbildung

ISSN 1424-3636

Themenheft Nr. 23: Visuelle Kompetenz

\section{Fotografieren im Sachunterricht - Ergebnisse aus einer Fallstudie}

Regula Fankhauser und Barbara Bader

\begin{abstract}
Im Unterschied zur dominierenden Stossrichtung innerhalb des medienpädagogischen Diskurses, welcher die Rezeption von Fotografien im schulischen Unterricht thematisiert, fokussiert der vorliegende Artikel auf das Fotografieren, das produktive Bilder-Machen von Schülerinnen und Schülern im Rahmen des Sachunterrichts. Anhand einer Berner Studie, die sich dem Zusammenhang von Repräsentationsmodus und Lernprozess widmete, geht der Artikel der Frage nach, wie sich fotografisch gelöste Beobachtungsaufgaben von sprachlich realisierten unterscheiden, welchen Aufschluss sie geben über die Lern- und Konstruktionsprozesse von Kindern und wie sie für das Lernen im Sachunterricht genutzt werden könnten. Der fallkontrastierende Vergleich macht deutlich, wie unterschiedlich, ja disparat die fotografischen Lösungswege sind, die die Kinder eingeschlagen haben, und wie wenig sie sich - im Unterschied zu den sprachlich arbeitenden Kindern - von Instruktionen lenken liessen. Dieser konstruktive Aspekt des Mediums Fotografie könnte im Sachunterricht deshalb - wenn gezielt eingesetzt und methodisch reflektiert - zur Realisierung eines mehr selbstgesteuerten Lernens dienen, in welchem der subjektive Blick auf die Welt als Teil der Wissenskonstruktion integriert würde.
\end{abstract}

\section{Theoretische Vorbemerkungen}

Überblickt man den medienpädagogischen Diskurs der letzten Jahre im Hinblick auf Bildkompetenz im Allgemeinen, so fällt auf, dass quasi als Subtext unterschiedlicher Fragestellungen zwei Zielsetzungen verfolgt werden: zum einen ein eher technischer Zugang, bei dem das Handling visueller Medien im Vordergrund steht, und zum andern ein ideologiekritischer Ansatz, welcher die kritische Decodierung bildlich formulierter Botschaften zum Ziel hat. Und wiewohl in den meisten theoretischen Arbeiten das Zusammenspiel von Produktion, Rezeption und Reflexion betont wird, so fokussieren die Modellierungen vorzugsweise den Rezeptionsaspekt - nicht selten dabei Kompetenzmodelle aus der Semiotik und der Bildwissenschaft aufnehmend (Posner 2003; Scholz 1998), welche Stufenmodelle des Bildverstehens vorschlagen, die letztlich auf die Hermeneutik und die Ikonologie zurückgehen. 
Bildkompetenz wird also mehrheitlich als Bildverstehen thematisiert, dessen Tiefe mit einem ideologiekritischen Zugriff aufs Bild korreliert. So plädiert beispielsweise Sachs-Hombach (2005) für ein Schulfach «Visuelle Medien», in dem Bildkompetenz als grundlegende Kulturtechnik verstanden und gefördert werden sollte und zwar im kantischen Sinne als Bildkritik, d.h. als kritisch-reflexive Auseinandersetzung mit den Möglichkeiten und Grenzen des Bildeinsatzes. Ganz ähnlich Reichenbach (2010); auch er spricht von einer Kulturtechnik, deren zentrales Moment er - wiederum im Rückgriff auf Kant - als ästhetische Urteilskompetenz versteht, welche ein «Studieren, also Bemühen» voraussetzt und statt Fähigkeiten vielmehr «Tugenden des Sehens» erfordert (ebd., 798).

Gemeinsam ist diesen Ansätzen nicht nur die - oft implizite - Priorisierung des Rezeptionsaspektes, sondern - damit verbunden - der Primat der Sprache. Analytisches Instrument einer kritisch-dekonstruierenden Bildlektüre ist die Begriffssprache und die diskursive Argumentation. Und gemeinsam ist diesen Ansätzen vielleicht auch ein bestimmter Traditionszusammenhang, in dem sie sich nolens volens verorten: demjenigen nämlich, der im Bild schon immer das Anarchische gesehen hat, welches das Wort in seinem Geltungsanspruch bedroht.

Dieser Sachverhalt, der hier sehr allgemein, entsprechend grobrasterig und rhetorisch zugespitzt zusammengefasst worden ist, lässt sich auch im Hinblick auf die Fotografie nachzeichnen. Vielleicht sogar in verschärftem Masse. Denn: gilt für die Fotografie nicht par excellence, was für das Bild unterschwellig immer schon galt und seinen fast magischen Status ausmacht (Boehm, 1994): die Verheissung nämlich, dass in ihm die Realität unmittelbar festgehalten und eingefangen werden kann? Die Kehrseite der Verheissung jedoch ist der Verdacht. Der Verdacht, dass das fotografische Bild «lügt», uns ein manipuliertes Versatzstück der Wirklichkeit präsentiert, ohne dass wir der Täuschung gewahr würden. Dieser Manipulationsverdacht, der dem fotografischen Bild anhängt, begünstigt seinerseits wieder das Weiterleben der Verheissung. Denn er stützt sich auf den Glauben, dass das fotografische Bild, wenn nicht manipuliert, uns die Wirklichkeit so zu sehen gäbe, wie wir sie tatsächlich mit unseren eigenen Augen sehen würden (Böhme 2004). Dass dem nicht so ist, ist mit einem Hinweis auf die Gesetzmässigkeiten des Sehens, beispielsweise dessen Zentriertheit und Randlosigkeit zu belegen. Dass wir trotzdem in unserem alltagspraktischen Umgang mit der Fotografie immer wieder auf diesem Glauben aufbauen, zeigt nur, wie umfassend das wissenschaftliche Ideal vom eindeutigen Abbilden auch die sinnlich-phänomenale Erfahrungswelt durchdrungen hat und zum Common Sense geworden ist.

Diesem alltagspraktischen Common Sense halten wir theoretisch zwei Prämissen entgegen, die von anderer Seite an anderer Stelle ausführlich entwickelt und begründet worden sind. Nämlich erstens: Es gibt keine eindeutige Art, wie wir die Realität sehen (Böhme 2004, 119). Und zweitens: Fotografien konstruieren Formen 
einer angenommenen Wahrheit des Sichtbaren (Stiegler 2009, 23). Den schulischen Unterricht im Auge, kommen wir zu folgenden Schlussfolgerungen, die wir einstweilen als Fragen formulieren: müsste nicht gerade das Fotografieren, d. h. das Bilder-Machen, Aufschluss darüber geben, wie Kinder und Jugendliche die Realität sehen? Welche Annahmen über die Welt sie treffen, wie sie ihr Wissen über die Welt konstruieren? Und welche Rolle der subjektive Blick auf die Welt für den Wissenserwerb überhaupt spielen könnte?

Diese Schlussfolgerungen als Hypothesen zu verfolgen, bedeutet medienpädagogisch eine Verschiebung des Interesses: weg vom Rezeptionspol, hin zum Produktionspol, weg vom Bilder-Lesen, hin zum Bilder-Machen. Dies impliziert ein Abrücken von der ideologiekritischen Frage nach der Wahrheit und dem «richtigen» Sehen und damit auch ein Abrücken vom Primat der Sprache, in der das jeweils Richtige verankert wird.

Stattdessen ginge es nunmehr darum, das So-und-so-Sehen von Schülerinnen und Schülern in seiner Interessegeleitetheit zum Thema zu machen; zu untersuchen, inwiefern sich diese individuellen Ansichten, diese unterschiedlichen Rahmungen von Wirklichkeit in den Fotografien von Schülerinnen und Schülern materialisieren, verlangt doch die Kamera ein Formulieren, Aufzeichnen, Sichtbarmachen (Kunz u. Stadler 2010). Und schliesslich zu fragen, wie wichtig dieses individuelle Sehen, dieser nicht-begriffliche Zugriff auf die Wirklichkeit für das Verstehen und das Lernen sein könnte. Fotografie als Quelle für die Lern- und Unterrichtsforschung also - im Folgenden soll dafür ein Beispiel vorgestellt werden.

\section{Eine Fallstudie}

In der Fallstudie «lch mach' mir ein Bild - Ästhetische Lehr- und Lernformen im Sachunterricht» (2010-2012, PHBern) wurde mit dem Titel und dessen Metaphorik eine doppelte Untersuchungsthematik aufgefaltet. "Ich mach' mir ein Bild»: Im eigentlichen Wortsinn ist damit das Herstellen von Bildern gemeint; im uneigentlichen dagegen die Rolle der bildhaften Anschauung beim Aufbau von Wissen und Verstehen.

In unserer Studie gingen wir der Verschränkung dieser beiden Wortbedeutungen nach. Das Untersuchungsinteresse betraf also den Zusammenhang zwischen Repräsentationsmodus und Lernprozess, welchen wir über einen Vergleich zwischen sprachlich arbeitenden und bildlich arbeitenden Lernenden zu erhellen hofften. Was für ein Bild würden sich Schülerinnen und Schüler von einem bestimmten Lerngegenstand machen, wenn sie sich diesen Lerngegenstand bildlich erschliessen mussten?

Zur Beantwortung dieser Frage entwickelten wir ein unterrichtliches Setting, das hier kurz skizziert werden soll. 
In einer fünften Klasse einer Berner Agglomerationsgemeinde wurde im Rahmen des NMM-Unterrichts (Sachunterricht) den Schülerinnen und Schülern die Aufgabe gestellt, ihren geografischen Nahraum, d. h. ihre Wohngemeinde, zu erkunden und nach bestimmten, vorgegebenen Kriterien zu beschreiben. Die eine Schülergruppe sollte dabei mit Papier und Bleistift vorgehen, die andere bekam hierzu einen Fotoapparat ausgehändigt. Abgesehen von den beiden unterschiedlichen Medien oder Repräsentationsmodi, durch die sich der Auftrag in den beiden Gruppen unterschied, lautete die Aufgabe für alle gleich. Sechs Stationen im Umkreis des Schulhauses (Diameter $500 \mathrm{~m}$ ), an denen Beobachtungen gesammelt werden sollten, waren vorgängig von der Lehrperson ausgewählt worden. Das gesammelte Material - hier sprachliche Notizen und Textfragmente, dort Fotos - sollte anschliessend in Partnerarbeit zu einem Plakat verarbeitet werden, welches neben einer Präsentation der Stationen auch einen zusammenfassenden, syntheseartigen Text zum Titel «Typisch Zollikofen» enthalten sollte. Die Partnerarbeit von acht ausgewählten Kindern, sogenannten Fokuskindern, wurde videografiert. Die acht Fokuskinder wurden anhand von vorgängig in einer Vorstudie erhobenen Daten - Zeichnungen und Texte - ausgewählt und zu je vier auf die beiden Gruppen, nämlich die Sprachgruppe und die Fotogruppe verteilt. Im Anschluss an die Unterrichtssequenz wurden sowohl die erstellten Schülerarbeiten wie der Arbeits- und Lernprozess in themenzentrierten Leitfadeninterviews mit den Fokuskindern besprochen. Die triangulierten Daten wurden inhaltsanalytisch ausgewertet, in Einzelfallbeschreibungen zusammengezogen und mit Hilfe von Fallkontrastierungen entlang der Auswertungskategorien in Strukturgeneralisierungen überführt. Wichtiges methodisches Moment war dabei immer wieder ein Verfahren, das wir als «Clustering» bezeichnen: das Zusammenführen von unterschiedlichen Daten - visuellen und sprachförmigen - zu Clustern, in welchen sich die Daten wechselseitig erhellen und dadurch neue Erklärungsmuster gefunden werden konnten.

Reflexiv betrachtet muss das Unterrichtssetting, das den Kern des Forschungsdesigns abgab, als relativ stark strukturiert bezeichnet werden. Zum einen waren die Stationen, an denen sich die Schülerinnen und Schüler umsehen sollten, vorgegeben. Zum andern waren die Beobachtungen durch eng geführte Fragen angeleitet. Instruktion gab also ein Raster vor, innerhalb dessen sich die Kinder ihren Lebensraum und ihr alltägliches Umfeld erschliessen sollten. Die Zielsetzung der instruierenden Aufgabenstellung orientierte sich dabei am deutschschweizerischen Lehrplan: die Schülerinnen und Schüler sollten sich von ihrem geografischen Nahraum, in diesem Fall die Agglomerationsgemeinde Zollikofen, ein sachgerechtes Verständnis aufbauen können.

Welcher Art nun ist dieses Verständnis - und wie bildet es sich im fotografischen Repräsentationsmedium ab? Was zeigen die Fotos der vier Fokuskinder, die in die 
Fotogruppe eingeteilt wurden, und was resultiert aus dem Vergleich zwischen den Kindern der Fotogruppe und denjenigen der Sprachgruppe?

Im Folgenden werden die fotografischen Dokumente der vier Fokuskinder aus der Fotogruppe exemplarisch vorgestellt.

Jessicas Fotografien sind stark durch ihren persönlichen Blick und ihre subjektive Wahrnehmung bestimmter Orte im geografischen Nahraum geprägt; nicht selten bleibt für aussenstehende Betrachter unklar, was Jessica sah und ins Bild zu setzen beabsichtigte. Relativ konstante Motive sind Personen und atmosphärische Bildelemente. Zahlreiche Fotografien sind in Bewegung erstellt und unscharf, wodurch ihnen etwas Anekdotisches anhaftet. Dokumentarische und imaginäre Elemente scheinen sich zu vermischen, der Zufall wird bei der Betätigung des Drückers eine wesentliche Rolle gespielt haben. Schnappschuss und Zoom sind zwei Möglichkeiten, die das Fotografieren bietet und welche Jessicas Zugriff auf die Welt entgegenkommen. Dass sie am Fotografieren v. a. die Zoomfunktion begeistert, erwähnt sie explizit, es zeigt sich aber auch in ihren Fotos. Die Möglichkeit, schnell und spontan das an sich heranholen zu können, was sie interessiert oder grad anspringt, kennzeichnet für sie das Medium und macht es für sie interessant. Gleichzeitig entbindet es sie jedoch auch von einem konzentrierten und sorgfältigen Hinschauen und Konzipieren. Im Unterschied zum sprachlichen Repräsentationsmodus, in welchem Jessica ihre subjektive, ja feinstoffliche Wahrnehmung der Lebensumgebung sehr sensitiv in einer adäquaten erzählerisch-phantasievollen Form aufgehen lassen kann, bleibt ihr diese Möglichkeit im Medium Fotografie weitgehend verschlossen. Dies liegt vielleicht weniger am Medium an sich, sondern daran, dass ihr die dafür notwendige technologische und bildnerische Erfahrung fehlt. Gleichzeitig sind ihr ihre Fotografien inspirierende Erzählanlässe, welche sie im Interview bereitwillig wahrnimmt.
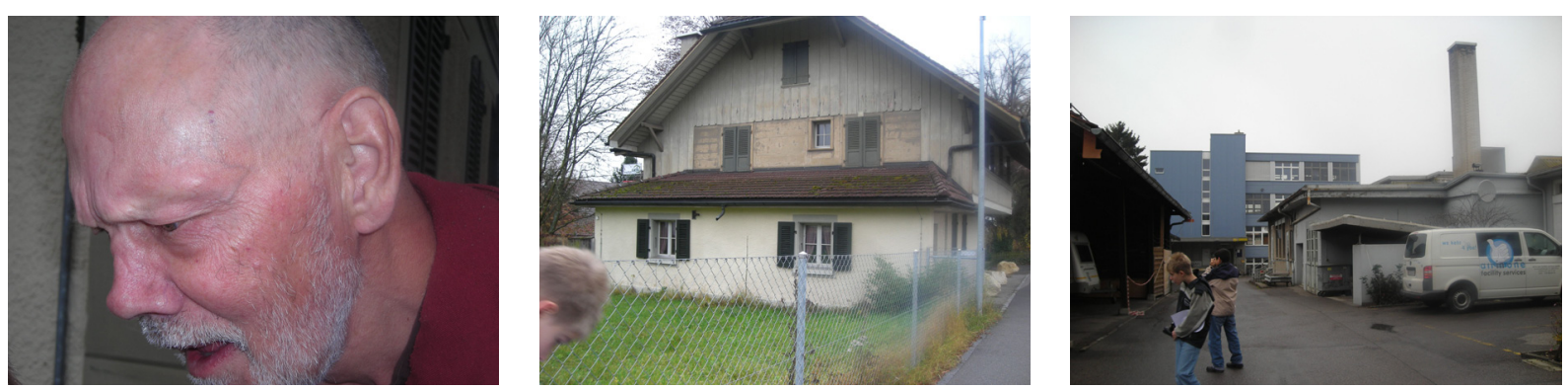

Abb.1-3: Jessica 

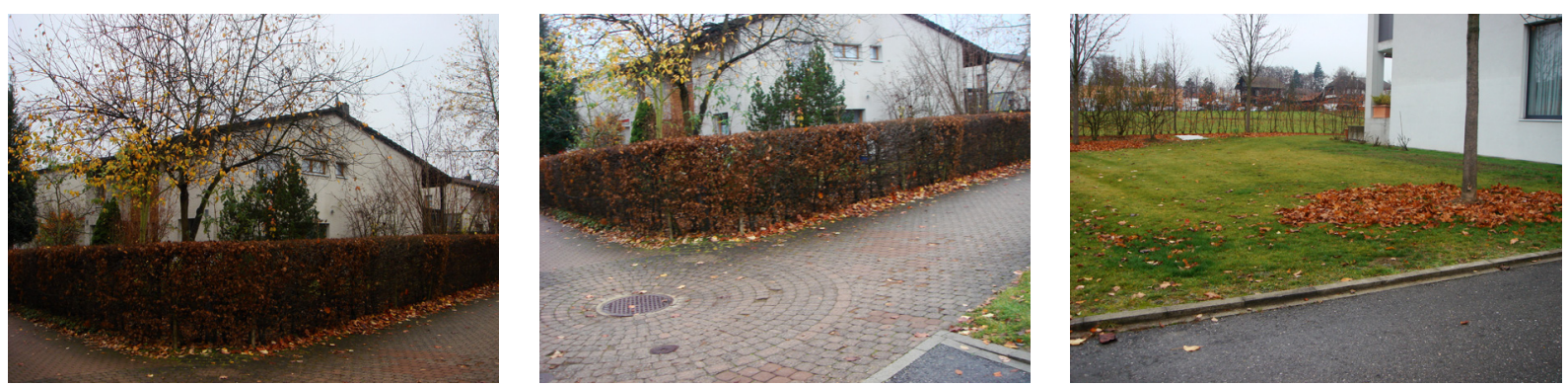

Abb. 4-6: Loris

Der bildliche Repräsentationsmodus entspricht Loris sowohl hinsichtlich seiner Motivation und seines Selbstkonzepts wie auch hinsichtlich seiner Fähigkeiten. Zeichnerische Konstruktionsaufgaben sind für ihn eine Herausforderung, der er sich hingebungsvoll stellt. Mit fotografischen Aufnahmen verfolgt er klare, zum Teil selbstauferlegte Bildabsichten, die er benennen und in unterschiedlichste Situationen und Medien transferieren kann. Als einziger der vier Fokuskinder bemüht sich Loris darum, die Fotografie als Medium zu gebrauchen, um auf die sprachlich formulierten Fragen der Aufgabenstellung zu antworten. Dass er sich hier von Qualitätsansprüchen leiten lässt, zeigt sich an wiederholten Versuchen, denselben Gegenstand abzubilden. In der Beurteilung seiner Bilder im Interview macht er deutlich, dass er zwischen verschiedenen Bildfunktionen unterscheiden und deren Qualität entsprechend der Funktion beurteilen kann. So macht er einen klaren Unterschied, ob ein Foto eine gegenstandsorientierte Funktion hat und also den Fremdreferenten möglichst informativ zeigen kann, oder ob seine Qualität eher bildimmanent oder selbstreferentiell zu bestimmen ist. Fotografie bedeutet für inn die Möglichkeit zum bewussten ins Bild hinein verlegten Zeigen. In der Wahl der Gegenstände und Bildausschnitte folgt Loris immer wieder einem ähnlichen Muster: er kombiniert Übersichtsdarstellungen mit Detailansichten. Sowohl das ganzheitliche Erfassen und Überblicken wie das Hervorheben von exemplarischen Einzelheiten eines Gegenstands ist ihm wichtig. Auf dem Plakat kombiniert er die Einzelfotos nach demselben Prinzip und erstellt dadurch so etwas wie semantische Netzwerke.

Loris kann seine Wahrnehmungserlebnisse im bildlichen Modus besser modellieren als im sprachlichen. Das Medium Fotografie kommt ihm in seinen Darstellungsabsichten entgegen; es bietet ihm Möglichkeiten, die in der Sprache weniger leicht zu ergreifen sind. Hier, wo er kognitive mit ästhetischen Vorgehensweisen verbinden kann, ist er lernfreudig und erlebt sein Tun als bedeutungsvoll. 

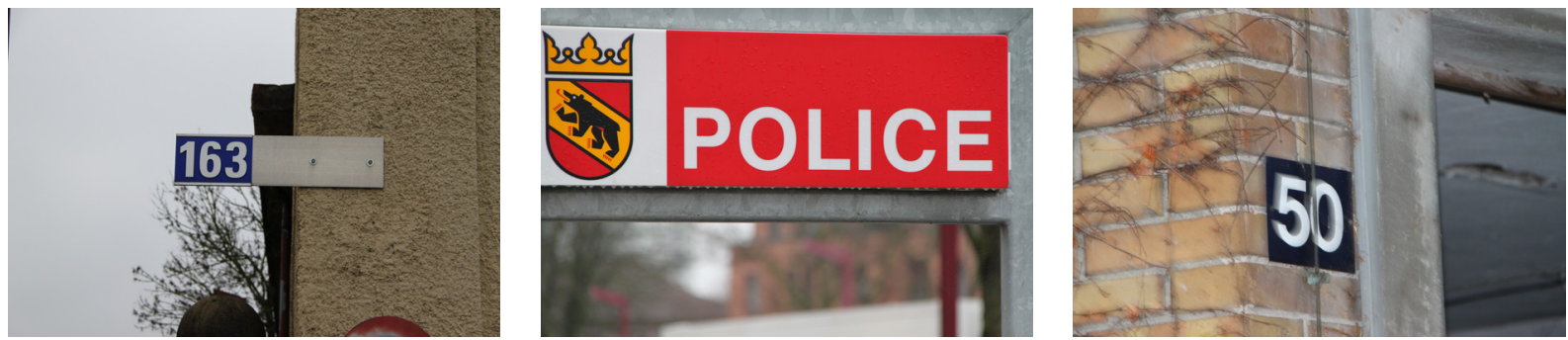

Abb. 7-9: Samuel

Während die Zeichnungen und Texte, die Samuel in der Vorstudie erstellt hat, rätselhaft anmuten, sind seine Fotografien von einer schon fast plakativen Deutlichkeit. Was auffällt, ist seine Vorliebe für Symbole, Schriftzeichen, Zahlen und Codes. Samuel bildet in seinen Fotos hauptsächlich Strassenschilder, Hausschilder und Verkehrsschilder ab. Dies ist sein bevorzugtes Motiv; seinen Lebensraum sucht er mit Hilfe des Objektivs nach diesen Anhaltspunkten ab. Was er finden will, ist eine figurale Oberflächenstruktur. Ob diese mehr als nur eine formal-ästhetische Bedeutung hat, ob sie also für Samuel auf eine darunter liegende Semantik verweist, ist nicht ausmachbar. Schriftzeichen, Ziffern, Symbole werden aus ihrem Kontext herausgelöst; ein Bezug zur Aufgabenstellung ist so nicht mehr herstellbar.

Wichtig scheint vielmehr die Etikettierung von einzelnen Gegenständen, deren Bezifferung und Kennzeichnung. Dies tritt gehäuft auf, wodurch seinen Fotografien ein ausgeprägter Belegcharakter anhaftet. Ebenfalls konstant ist die streng komponierte Bildstruktur, besonders die orthogonale Anordnung von Linien und Objekten sowie die Betonung des Bildzentrums.

Der Fotoapparat, so der Schluss, dient Samuel dazu, sein ganz persönliches Wahrnehmungs- und Darstellungsinteresse zu verfolgen und sich über Instruktionen, Erwartungen und schulische Ansprüche hinwegzusetzen. 

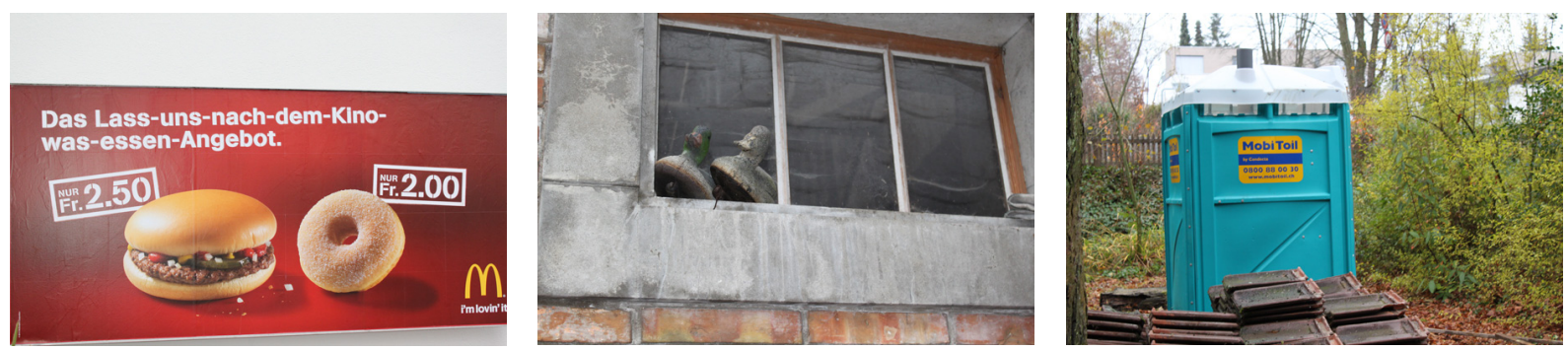

Abb. 10-12: Olaf

Olaf ist ein Macher - mit Eifer widmet er sich gestalterischen Aufgaben, bei denen er etwas in die Hand nehmen kann. Sein Zugriff auf die Welt ist sinnlich und direkt; die Fotografie ist für ihn ein ideales Medium, um seinen Neigungen nachgehen zu können. Diese sind oft leibsinnlicher oder ästhetischer Natur. Olaf fotografiert unterschiedlichste Motive, zum Teil aufgabenbezogen, zum Teil nicht. Seine Orientierung an leibsinnlichen Kriterien und an handfesten Tatsachen zeigt sich in seinen Bildgegenständen - einem Plakat mit einem Hamburger z. B., oder dem Klo der Bauarbeiter. Seine ästhetischen Interessen spiegeln sich nicht nur in entsprechenden Objekten, die er fokussiert, sondern auch in der Präsentation seiner Fotos, die er beim Ausschneiden mit gezeichneten unterschiedlichen Gesamtformen und Randmustern versieht, welche Bildinhalte oder Bildsprache der Fotos aufnehmen und verstärken.

$\mathrm{Ob}$ seine Fotos an die Aufgabenstellung anknüpfen, scheint Olaf dagegen nicht gross zu kümmern. Auf die Frage, mit welchen Fotos er nun eigentlich die Aufgabenstellung am besten umgesetzt habe, weicht Olaf aus, berichtet Anekdotisches und zeigt relativ beliebig auf ein Foto. Hier wird deutlich, dass sich Olaf kaum instruieren liess beim Lösen der Fotoaufgabe, sondern dass er die Möglichkeit wahrnahm, seinen spontanen Einfällen und Interessen nachzugehen und unvorhergesehene Lösungswege zu suchen. Ob dies allenfalls auch daran liegen könnte, dass Olaf in seiner Freizeit fotografiert und in seiner Fotopraxis von seinem familiären Umfeld bestärkt wird, bleibt unklar.

Insgesamt lassen sich seine Fotos als impulsive visuelle Echos auf Vorhandenes bezeichnen. Sie zeugen von einer noch etwas richtungslos scheinenden Sensibilität für seine Lebensumgebung und die eigene Präsenz darin. So hat Olaf ein Auge für Schönes - was ihm gefällt, fängt er fotografisch ein - in Bildern, die ihm erklärtermassen gefallen. 


\section{Schlussfolgerungen}

Fassen wir zusammen: Die Bildlösungen, die die Schülerinnen und Schüler der Fotogruppe wählen, verfolgen eigenständige, disparate Ansätze. Jessica fokussiert Menschen und lässt sich vom Moment leiten. Ihre Schnappschüsse evozieren persönlich erlebte Geschichten und dienen ihr später als Ausgangspunkt für Anekdoten. Samuel sucht figurale Zeichen, Schriftzüge und Ziffern, die er in Nahaufnahmen dekontextualisiert und prominent ins Bild setzt. Und Olaf hat ein Auge für Objekte, die an sinnliche Erfahrungen gebunden sind oder die ihm spontan gefallen.

Nur einer von ihnen, nämlich Loris, setzt das Medium als Lernwerkzeug ein, so wie es in der Aufgabenanlage vorgesehen war. Nur Loris versucht, mit Hilfe des Objektivs genau und zielgerichtet zu beobachten und diese Beobachtungen in Form einer fotografischen Aufnahme zu dokumentieren. Die andern scheren aus der Instruktion aus - suchen ihre eigenen Wege, lassen sich von ihren Wahrnehmungsgewohnheiten und Interessen leiten und zeigen ihre Lebenswelt aus ihrer Perspektive.

In den Fotografien der Fokuskinder zeigt sich neben der Dokumentation der Agglomerationswirklichkeit und dem eigenen Lesen bzw. Erleben dieser Lebensumgebung auch, wie die Kinder das Gesehene fotografisch ins Bild setzen (Kunz 2001, 199). In diesem doppelten Zeigen des Was und des Wie zeigt sich indirekt etwas Drittes: ihr «cognitive style», d.h. die Art und Weise, wie sie im und mit dem Bild Informationen verarbeiten - wahrnehmen, verknüpfen und abstrahieren - oder einfach gesagt, wie sie mit Bildern lernen (Gardner 2006, 05). Gerade weil die Schülerinnen und Schüler keine Einführung oder Instruktionsanweisung erhalten hatten, wie ihre fotografischen Antworten auf die ortsspezifischen Fragen zu erstellen seien oder auszusehen hätten, zeigten sich in der Kontrastierung der vier Fälle höchst unterschiedliche individuelle fotografische Arbeitsweisen bzw. Verarbeitungsmodi visueller Information (Glaser-Henzer et al. 2012, 101ff). Die Spannbreite reicht von rein belegenden Abbildungen über stark mit Empfindungen aufgeladenen Fotografien bis hin zu Aufnahmen, denen ein abstrakter, konzeptueller Abbildungsanspruch unterliegt. Im Binnenvergleich zeigen sich individuelle Präferenz und Stil, insbesondere in der Wiederholung, sei es auf der Ebene des Bildinhaltes, des Bildanlasses oder der Bildstruktur. Augenfällige Repetitionen, die bei allen Fokuskindern erkennbar sind, repräsentieren dabei manchmal spielerische Wiederholung, manchmal aber auch die wiederholte Suche nach einer prägnanteren fotografischen Formulierung in Form von Reihen und Clustern.

Zusammengenommen sind sie die sichtbare Spur von Denk- und Lernprozessen oder anders gesagt: Zeugen individueller «cognitive styles».

Zieht man nun den Vergleich mit der Sprachgruppe, d. h. mit den Schülerinnen und Schülern, die dieselbe Aufgabe mit Papier und Bleistift lösen mussten, so 
tritt das für die Fotografie herausgearbeitete Spezifikum noch deutlicher zum Vorschein. Die Dokumente der Sprachgruppe, es handelt sich um Aufgabenblätter mit Schülernotizen, variieren untereinander nur minim. Das textförmige Material ist sowohl bezüglich Inhalt wie bezüglich Form einheitlicher. Die Notizen, die sich die Schülerinnen und Schüler, angeleitet von den Fragen auf den Aufgabenblättern, machen, ähneln sich insgesamt relativ stark. Das inhaltliche Antwortspektrum ist begrenzt, die Schülerinnen und Schüler lassen sich überdies leicht durch die Frageformate instruieren. Ihre Antworten nehmen zumindest dort, wo die Aufgaben sorgfältig gelöst worden sind, auch grammatikalisch Bezug auf die Fragen und suchen nach einer formalen Passung.

Dagegen sind die Lösungen, die von der Fotogruppe präsentiert wurden, einerseits eigenständiger und individueller, andererseits aber auch weniger aufgabenbezogen als die textförmigen Lösungen. Die fotografisch arbeitenden Schülerinnen und Schüler konnten offensichtlich ihre ganz eigenen Wege gehen - diese haben sie aber stellenweise auch weit vom ursprünglich vorgesehenen und von der Lehrperson geplanten Ziel abgebracht. Die sprachlich arbeitenden Schülerinnen und Schüler dagegen bewegen sich stärker in den didaktisch vorbereiteten Pfaden und lassen sich durch die instruierende Aufgabenstellung lenken und anleiten. Sie nutzen das Lernangebot so, wie es vom didaktischen Setting her vorgesehen war. Der Schluss ist naheliegend: in der Fotogruppe verstärkt sich das von der Unterrichtsforschung herausgearbeitete generelle Problem der Kontingenz (Kade 1997; Meseth, Proske u. Radtke 2012). Unterrichten ist eine Praxis mit ungewissen Wirkungen; das Verhältnis zwischen Vermittlung und Aneignung ist kein kausales, sondern eines, welches auf Differenz beruht. Wie Schülerinnen und Schüler mit dem Angebot, das ihnen Lernumgebungen bieten, umgehen, ist offen (Lipowsky, 2006). Lernen ist ein konstruktiver Prozess, keine noch so gute Instruktion kann diesen vorwegnehmen oder ersetzen. Dass der Grad der Konstruktivität allerdings auch von der Lernumgebung, den Lernmedien und dem Repräsentationsmodus abhängt, hat unsere Studie deutlich machen können. Das Medium Fotografie und der bildliche Repräsentationsmodus scheinen den Lernenden in verstärktem Masse Möglichkeiten zu bieten, einerseits selbstgesteuerte, eigenständige Lernwege zu gehen und andererseits didaktisch intendierte Zielsetzungen zu ignorieren.

Dies mag zum einen mit mangelndem Methodenwissen im Umgang mit dem fotografischen Medium zu tun haben, das hier für einmal nicht dem Spass und dem Spiel, sondern dem Lernen dienen soll. Zum andern aber liegt dies auch im Medium selber begründet. Anders als im begrifflich definierenden oder gar mathematisch modellierenden Zugreifen auf die Wirklichkeit, wird in der Fotografie gerade nicht eine generell gültige Sichtweise gesetzt. Vielmehr werden - wie im Eingangskapitel erläutert - unterschiedliche Rahmungen von Wirklichkeit sichtbar. Dieser konstruktive Charakter des Mediums Fotografie könnte gerade im Kontext 
von Schule und Unterricht, speziell im Sachunterricht genutzt werden. Hier gilt nur zu oft, dass durch spezifisches Fragen und besonders dann durch die Verschriftlichung unterschiedliche und individuelle Aneignungsoptionen eingeschränkt und im Hinblick auf gesichertes Wissen vereindeutigt werden (Meseth 2010, 230). Der gezielte Einsatz des Fotoapparates könnte hier ein pluralistischeres und individuumszentriertes Lernen unterstützen und gleichzeitig auch den Konstruktionscharakter von Wissen und Wissensdarstellung anhand des eigenen Produktionsprozesses erfahrbar machen.

Damit die Balance zwischen Instruktion und Konstruktion jedoch gehalten werden kann und Fotografieren nicht nur als willkommener Ausgleich zum ansonsten sprachlich dominierten Unterricht erlebt wird, müsste solides Methodenwissen zur fotografischen Bildsprache und ihren praktischen Einsatzmöglichkeiten ausserhalb des Kunstunterrichts vermittelt und eingeübt werden. Ein Desiderat, dessen didaktisch-methodische Verwirklichung unseres Erachtens noch in weiter Ferne liegt.

\section{Literatur}

Boehm, Gottfried. 1994. «Die Bilderfrage.»In Was ist ein Bild?, hrsg. v. Gottfried Boehm, 325-343. München: Wilhelm Fink Verlag.

Böhme, Gernot. 2004. Theorie des Bildes. München: Wilhelm Fink Verlag.

Gardner, Howard. 2006. Multiple Intelligences. New York: Basic Books.

Glaser-Henzer, Edith, Ludwig Diehl, Luitgard Diehl Ott und Georg Peez. 2012. Zeichnen: Wahrnehmen, Verarbeiten, Darstellen. München: kopaed.

Kade, Jochen. 1997. "Vermittelbar/Nicht-Vermittelbar: Vermitteln: Aneignen im Prozess der Systembildung des Pädagogischen.»In Bildung und Weiterbildung im Erziehungssystem, hrsg. v. Dieter Lenzen u. Niklas Luhmann, 30-70. Frankfurt/M.: Suhrkamp.

Kunz, Ruth und Brigitte Stadler. 2010. Unterwegs: Fotografische Bildfindungsprozesse von Jugendlichen in urbanen Lebenswelten. Bd. 3. Bildfindungsprozesse. Zürich: Pädagogische Hochschule Zürich.

Lipowsky, Frank. 2006. «Auf den Lehrer kommt es an.» Zeitschrift für Pädagogik 51 (Beiheft 52): 47-65.

Meseth, Wolfgang, Matthias Proske und Frank-Olaf Radtke. 2012. «Kontrolliertes Laissez-faire: Auf dem Weg zu einer kontingenzgewärtigen Unterrichtstheorie.» Zeitschrift für Pädagogik 58 (2): 223-241.

Posner, Roland. 2003. «Ebenen der Bildkompetenz.»In Was ist Bildkompetenz? Studien zur Bildwissenschaft, hrsg. v. Klaus Sachs-Hombach, 17-24. Wiesbaden: Deutscher Universitäts-Verlag.

Reichenbach, Roland und Nicolaj van der Meulen. 2010. "Ästhetisches Urteil und Bildkompetenz.» Zeitschrift für Pädagogik 56 (6): 795-805. 
Sachs-Hombach, Klaus. 2005. «Plädoyer für ein Schulfach 〈Visuelle Medien`.» Filmforschung und Filmlehre (Themenheft zu IMAGE 2): 1-5.

Scholz, Oliver. 1998. «Was heisst es, ein Bild zu verstehen?» In Bild - Bildwahrnehmung - Bildverarbeitung: Interdisziplinäre Beiträge zur Bildwissenschaft, hrsg. v. Klaus Sachs-Hombach u. Klaus Rehkämper, 105-118. Wiesbaden: Deutscher Universitäts-Verlag.

Stiegler, Bernd. 2009. Montagen des Realen: Photographie als Reflexionsmedium und Kulturtechnik. München: Wilhelm Fink Verlag. 